\section{Aspectos epidemiológicos do dengue no Município de São Luís, Maranhão, Brasil, 1997-2002}

\author{
Epidemiological characteristics of dengue \\ in the Municipality of São Luís, Maranhão, \\ Brazil, 1997-2002
}

Vicente Silva Gonçalves Neto 1 José Manuel Macário Rebêlo 2

\footnotetext{
1 Mestrado em Saúde e Ambiente, Universidade Federal do Maranhão, São Luís, Brasil.

2 Departamento de Biologia, Universidade Federal do Maranhão, São Luís, Brasil.

Correspondência J. M. M. Rebêlo Departamento de Biologia, Universidade Federal do Maranhão. Praça Madre Deus 2 São Luís, MA 65025-560, Brasil. macariorebelo@uol.com.br
}

\begin{abstract}
A total of 12,008 cases of dengue were reported from 1997 to 2002, with peaks in 1997 (35.8\%) and 1998 (45.8\%). The disease predominated in the 15-49-year age group (72.2\%) and during the rainy season (83.3\%), showing a positive correlation with the amount of rainfall $(r=0.84)$ and relative humidity ( $r=0.76)$ and a negative correlation with temperature $(r=-0.78)$. There was no significant difference $\left(\chi^{2}=2.319 ; p>\right.$ $0.05)$ in the number of cases in males (49\%) and females (51\%). Dengue was detected in all seven health districts: Tirirical (27\%), Coroadinho (17\%), Bequimão (16\%), Cohab (14\%), ItaquiBacanga (11\%), Centro (8\%), and Vila Esperança (7\%). The building infestation rate ranged from 1.01 to 7.15 , and the Breteau index ranged from 1.10 to 8.18 . The most frequent mosquito breeding sites were metal barrels or similar recipients (80.0\%), followed by plant vases (4.7\%), bottles, cans, and plastics (3.6\%), tires (3.3\%), water tanks (2.7\%), and others (5.7\%). In the case of Aedes aegypti, 134,631 positive water deposits were detected, with a predominance of type $B$ (80.0\%), followed by types $C$ (4.7\%), E (3.6\%), and $A$ (3.3\%).
\end{abstract}

Dengue; Aedes; Local Health Systems

\section{Introdução}

O dengue constitui uma endemia em ascendência no Brasil. Basta verificar que, em 1980, apenas 12 municípios estavam infestados pelo Aedes aegypti e, ao fim de 1998, esse número aumentara para aproximadamente 2.910 1. Em 2001, 3.587 municípios das 27 unidades federadas encontravam-se infestados e a transmissão da infecção já ocorria em 2.262 municípios de 24 Estados 2.

Vários fatores concorreram para o agravamento da situação e dispersão do vetor: condições ambientais precárias dos grandes centros urbanos; aumento considerável no fluxo migratório; escoamento de produtos/mercadorias; condições de umidade e temperatura favoráveis à proliferação dos vetores; e a pouca efetividade das ações de controle vetorial implementadas, que muitas vezes sofrem solução de continuidade 1 , entre outros.

Entre os anos de 1982 e 1997, a Região Nordeste contribuiu com cerca de 502.772 casos de dengue, ou 54,00\% das notificações ocorridas no país; no Maranhão foram notificados, no mesmo período, 14.190 casos, representando $2,83 \%$ dos casos nordestinos 3 . No período decorrido entre 1998 e 2001, os casos nordestinos aumentaram para 652.448 registros, correspondendo a $46,30 \%$ dos casos nacionais, enquanto o Maranhão nesse mesmo período notificou 27.884 casos, ou 4,30\% das notificações do Nordeste 2,4 . 
Em 1996 aconteceu a primeira epidemia na ilha de São Luís, com 4.641 casos notificados. Nesse ano realizou-se um inquérito soroepidemiológico na ilha, estimando-se que $41,40 \%$ da população estudada se encontrava sensibilizada pelo DEN-1. A epidemia prosseguiu até 1998, havendo dificuldade para confirmação dos casos em laboratório 5. Em 2001 foi isolado o sorotipo DEN-2 6 .

O Ae. aegypti foi introduzido em São Luís em 1969, mas só chamou a atenção dos órgãos de saúde no ano de 1995, quando se detectaram os primeiros casos de dengue clássica no bairro da Cohab-Anil. Por iniciativa do Ministério da Saúde, diante da gravidade da situação, foi montada uma estratégia de combate ao vetor com base no Plano Diretor de Erradicação do Ae. aegypti. Mediante convênio firmado entre o Ministério da Saúde e a prefeitura de São Luís, garantiu-se a implementação das atividades direcionadas ao combate do Ae. aegyp$t i$, que vêm se desenvolvendo desde o ano de 1995, sem que, entretanto, obtenham-se resultados satisfatórios constantes 7 .

Em 2001 a Fundação Nacional de Saúde/ Ministério da Saúde e a Organização Pan-Americana da Saúde elaboraram o Plano de Intensificação das Ações de Controle da Dengue, com o objetivo de otimizar os pontos positivos já consolidados com referência ao controle da doença e seu vetor, bem como, através do Plano Nacional de Controle da Dengue, de 2002, buscar o envolvimento de todas as instâncias que compõem o Sistema Único de Saúde, enfatizando o papel da atenção básica no controle da dengue 8 .

Diante desse quadro e conhecendo-se a complexidade e a extensão do problema da dengue em São Luís, resolveu-se realizar este trabalho com o objetivo de estudar a situação epidemiológica da doença, dando a conhecer a distribuição de casos de acordo com distritos, anos, meses, faixa etária e sexo dos pacientes, índices de infestação predial e de Breteau por $A e$ des, e número de depósitos positivos para $A e$. aegypti e Ae. albopictus.

\section{Material e métodos}

\section{Área de estudo}

O Município de São Luís está situado na ilha de São Luís, Estado do Maranhão, localizado a 2031' LS e 4418' LW, e a uma altitude média de
32 metros. A área é de $822,1 \mathrm{~km}^{2}$, que corresponde aproximadamente a $0,24 \%$ do território do estado. Atualmente encontra-se dividido em sete distritos sanitários (DS): Centro, Itaqui-Bacanga, Coroadinho, Cohab, Bequimão, Tirirical e Vila Esperança. No ano de 2000, a população do município era de 855.442 habitantes, dos quais 822.935 urbanos (381.019 masculinos; 441.916 femininos) e 32.507 rurais (16.676 masculinos e 15.831 femininos) 9 .

O clima é o tropical quente e úmido, com duas estações: a chuvosa (janeiro a junho), com precipitação pluviométrica média de $1.954 \mathrm{~mm}$, e a de estiagem (julho a dezembro). A temperatura varia entre $28^{\circ} \mathrm{C}$ e $30^{\circ} \mathrm{C}$. O município é cortado por rios, estuários, córregos, igarapés e riachos.

\section{Metodologia}

Trata-se de um estudo descritivo do tipo ecológico misto, sendo utilizadas as seguintes variáveis: (a) espacial (município); (b) temporal (ano, mês e estações); (c) demográficas (sexo e faixa etária); (d) meteorológicas (índice pluviométrico, umidade relativa do ar e temperatura); (e) distribuição da doença; e (f) índices de infestação do Aedes. Utilizaram-se dados secundários referentes ao período de 1997 a 2002 das seguintes fontes: relatórios da Secretaria Municipal de Saúde do Município de São Luís 6,7, contendo informações epidemiológicas e de expansão da doença, organizadas em tabelas e gráficos; Fundação Instituto Brasileiro de Geografia e Estatística (população); e Sistema de Informação de Febre Amarela e Dengue (FAD), Fundação Nacional de Saúde. Os dados meteorológicos foram obtidos na Estação de Meteorologia do Destacamento de Proteção ao Vôo da Força Aérea Brasileira, situada no município de São Luís. A classificação dos depósitos está de acordo com o FAD: A - pneu; B - tambor/ tanque/tina/tonel/depósito de barro; $\mathrm{C}$ - vaso de planta; D - material de construção/peça de carro; E - garrafa/lata/plástico; F - poço/cisterna/cacimba; G - caixa d'água; $\mathrm{H}$ - recipiente natural; I - outros; J - armadilhas; $\mathrm{K}$ - pool.

\section{Análises estatísticas}

Para a realização dos testes estatísticos, utilizou-se o programa Bioestat 2.0. A correlação de Pearson foi utilizada para analisar a variação dos dados meteorológicos em relação à freqüência de casos da doença durante os meses, visando a identificar um padrão de variação 
conjunta entre essas variáveis. Utilizou-se ainda o teste do qui-quadrado $\left(\chi^{2}\right)$ de independência para analisar as diferenças na proporção de casos por sexo, faixa etária e estação do ano. As diferenças foram consideradas significativas quando a probabilidade (p) do erro foi inferior a $5 \%(\mathrm{p}<0,05)$.

\section{Resultados}

\section{Distribuição dos casos de dengue de acordo com ano, faixa etária e sexo}

Foram notificados 12.008 casos da doença, dos quais 35,80\% correspondentes ao ano de 1997 e $45,80 \%$, a 1998 , representando um aumento de $10,00 \%$. Nos anos seguintes houve notável diminuição do número de casos, tendo sido notificados apenas $18,4 \%$ (Tabela 1 ).

A doença predominou na faixa etária de 1549 anos, com 8.668 casos $(72,20 \%)$, contra 3.340 $(27,80 \%)$ nas demais faixas combinadas (Tabela 2). Essa diferença foi estatisticamente significativa $\left(\chi^{2}=230,766 ; 5\right.$ GL; $\left.\mathrm{p}=0,000 ; \mathrm{p}<0,05\right)$.

No total foram registrados 5.885 casos no sexo masculino, representando 49,00\%, e 6.123 $(51,00 \%)$ no feminino (Tabela 3$)$. Não houve diferença significativa no número total de casos entre ambos os sexos $\left(\chi^{2}=2,319 ; \mathrm{p}=0,1278 ; \mathrm{p}<\right.$ $0,05)$. Entretanto, quando a análise leva em consideração cada ano, verifica-se que há diferença significativa somente no ano de $1999\left(\chi^{2}=\right.$ 4,938; $\mathrm{p}=0,0263 ; \mathrm{p}<0,05)$.

\section{Distribuição sazonal da dengue}

Observou-se maior freqüência de casos na estação chuvosa $(83,80 \%)$, em detrimento do período de estiagem $(16,20 \%)$ (Tabela 1). Entretanto, a variação na casuística foi maior no período chuvoso, registrando-se $3,20 \%$ dos casos em janeiro e $24,40 \%$, em março. Na estação de estiagem essa diferença foi menor, variando de $0,70 \%$ (dezembro) para $7,00 \%$ (julho). Houve uma correlação positiva, ao longo dos anos, com a precipitação pluviométrica $(r=0,8415)$ e a umidade relativa $(r=0,7606)$, e negativa com a temperatura $(r=-0,7838)$.

\section{Distribuição dos casos de dengue por distritos sanitários}

No total, os sete distritos resultaram positivos, prevalecendo o do Tirirical $(27,00 \%)$, seguido por Coroadinho (17,00\%), Bequimão $(16,00 \%)$, Cohab (14,00\%), Itaqui-Bacanga $(11,00 \%)$, Centro $(8,00 \%)$ e Vila Esperança $(7,00 \%)$.
Índices de infestação predial e de Breteau por Ae. aegypti e depósitos e imóveis tratados

Analisando a Tabela 4, observa-se que o índice de infestação predial (IIP) variou no período estudado, com os maiores registros nos três primeiros anos, diminuindo acentuadamente em 2000, elevando-se substancialmente em 2001 e decrescendo em 2002. O mesmo padrão pode ser observado em relação ao índice de Breteau (IB). No total, foram tratados cerca de 3.701.153 imóveis e 9.921.675 depósitos.

\section{Número de depósitos positivos para Ae. aegypti e Ae. albopictus encontrados em imóveis}

Foram encontrados 134.631 e 3.534 depósitos positivos, respectivamente para Ae. aegypti e Ae. albopictus (Tabela 5). Os depósitos com maiores freqüências de Ae. aegypti foram: B (80,00\%), C $(4,70 \%), \mathrm{E}(3,60 \%)$, A (3,30\%) e I (2,80\%). Para Ae. albopictus os depósitos mais importantes foram os do tipo B (52,10\%), E (14,20\%), A (13,30\%), I $(7,40 \%)$ e C $(5,60 \%)$.

\section{Discussão}

Os dados obtidos neste estudo mostram que a doença grassou principalmente nos primeiros dois anos, com o coeficiente de incidência (CI) por 100 mil habitantes de 535,6 em 1997, seguindo-se um agravamento em 1998 (CI =671,0). Vale lembrar que, até então, os dados disponíveis indicavam apenas a presença de DEN-1. A circulação viral foi se expandindo ao longo desses anos nos distritos, incorporando novos bairros e novo contingente populacional. Em 1997, o pico concentrou-se em maio, pois a circulação viral foi se estabelecendo nos primeiros meses e a inexistência de imunidade de grupo supostamente propiciou a "explosão" da epidemia. O mesmo pode ter acontecido no ano de 1998, à medida que a população de novos bairros ia sendo atingida, mas não se pode refutar a possibilidade da circulação simultânea do DEN2 nesse último ano, como ocorreu em municípios ao sul do Maranhão (Açailândia, Balsas e Chapadinha) 5 e em outras cidades brasileiras, como Rio de Janeiro; Salvador, Bahia; e Fortaleza, Ceará 10,11,12. Tal quadro inspira cuidados redobrados para prevenir possíveis situações de febre hemorrágica da dengue (FHD), requerendo, portanto, a implementação de medidas de vigilância e controle.

A partir de 1999 observou-se uma diminuição nas taxas de incidência da doença, possi- 
Casos notificados de dengue clássica e dados meteorológicos por mês/ano e estação, no Município de São Luís, Maranhão, Brasil, $1997-2002$.

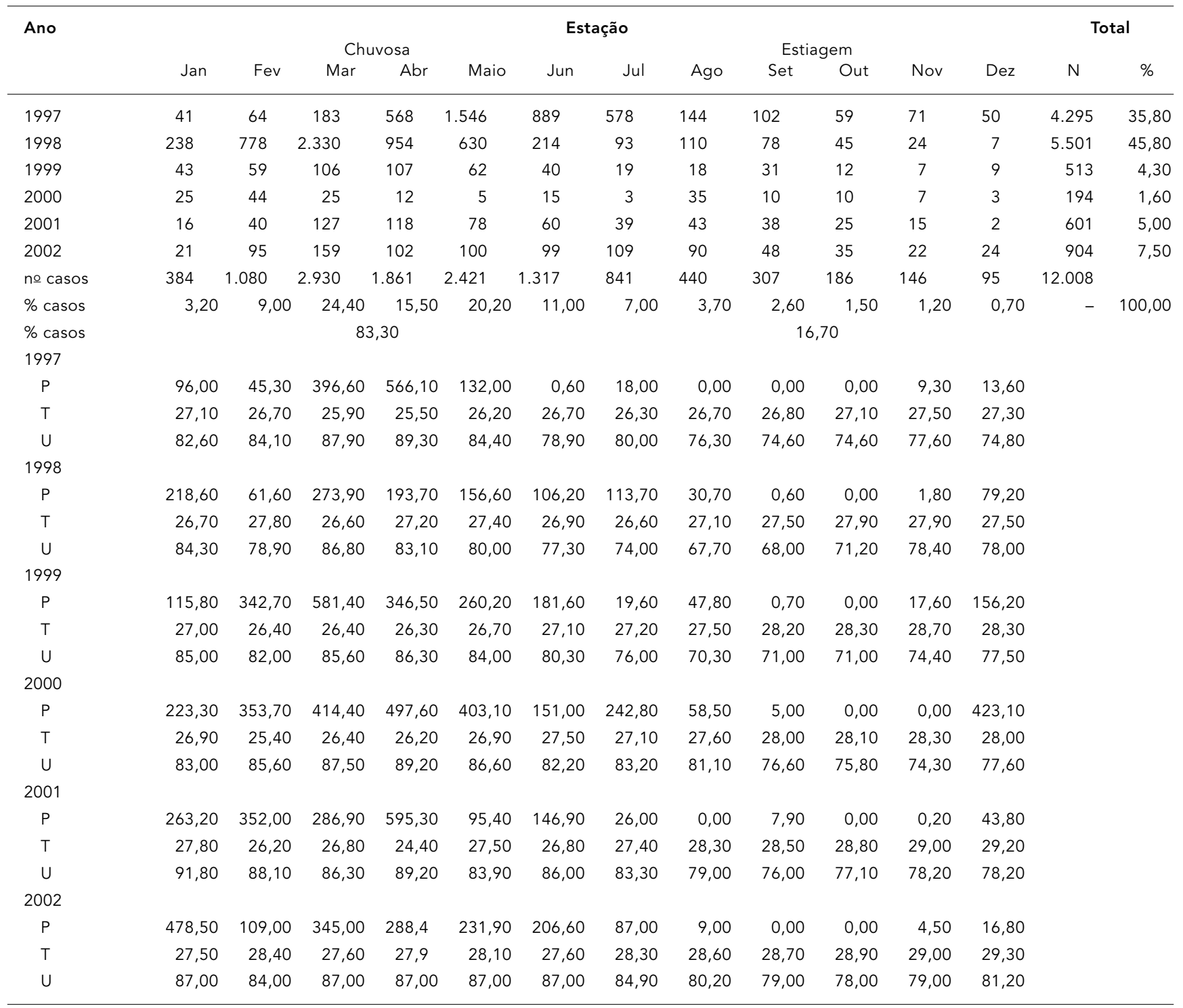

Fonte: Secretaria Municipal de Saúde do Município de São Luís 6,7; P = precipitação pluviométrica;

$\mathrm{T}$ = temperatura; $\mathrm{U}=$ umidade relativa; $\mathrm{N}$ = número de casos de dengue clássica notificados.

velmente em decorrência do estabelecimento de uma imunidade de grupo parcial, fator a ser levado em conta nessa situação 10 . Por outro lado, não se podem descartar os problemas operacionais, como falhas no sistema de informações, pouco interesse dos profissionais de saúde em efetuar a notificação e deficiências estruturais na assistência das unidades do Sistema Único de Saúde, fazendo com que a população, já sabendo como tratar a dengue clássica, não procure atendimento em tais unidades.

Em 2001, após considerável queda no número de casos de dengue nos dois anos ante- riores, foi isolado o sorotipo DEN-2, o que, a princípio, explica o leve pico no número de casos em comparação com o ano anterior, corroborando o que outros autores observaram em outras cidades de grande porte como São Luís 10,11,12. No ano de 2002 notificaram-se quatro casos de FHD, com um óbito, coincidindo com a circulação do DEN-3 6, causando um estado de alerta, como costuma acontecer em presença de novo sorotipo ${ }^{13}$, população sensibilizada e alto índice de infestação do Ae. aegypti.

Este estudo mostrou a baixa freqüência de FHD, corroborando as observações de Vascon- 
Tabela 2

Casos notificados de dengue clássica segundo a faixa etária no Município de São Luís, Maranhão, Brasil, 1997-2002.

\begin{tabular}{|c|c|c|c|c|c|c|c|c|c|c|c|c|}
\hline \multirow[t]{3}{*}{ Ano } & \multicolumn{10}{|c|}{ Idade (em anos) } & \multicolumn{2}{|c|}{ Total } \\
\hline & \multicolumn{2}{|c|}{$<5$} & \multicolumn{2}{|c|}{$5-14$} & \multicolumn{2}{|c|}{$15-49$} & \multicolumn{2}{|c|}{$>50$} & \multicolumn{2}{|c|}{ Ignorada } & & \\
\hline & $n$ & $\%$ & $n$ & $\%$ & $\mathrm{n}$ & $\%$ & $n$ & $\%$ & $n$ & $\%$ & $n$ & $\%$ \\
\hline 1997 & 150 & 3,50 & 687 & 16,00 & 3.033 & 70,60 & 378 & 8,80 & 47 & 1,1 & 4.295 & 100,00 \\
\hline 1998 & 159 & 2,90 & 523 & 9,50 & 4.236 & 77,00 & 556 & 10,10 & 27 & 0,5 & 5.501 & 100,00 \\
\hline 1999 & 11 & 2,20 & 61 & 11,90 & 369 & 71,90 & 72 & 14,00 & 0 & 0,0 & 513 & 100,00 \\
\hline 2000 & 1 & 0,50 & 18 & 9,30 & 158 & 81,40 & 17 & 8,80 & 0 & 0,0 & 194 & 100,00 \\
\hline 2001 & 45 & 7,50 & 134 & 22,30 & 363 & 60,50 & 60 & 10,00 & 0 & 0,0 & 601 & 100,00 \\
\hline 2002 & 105 & 11,60 & 199 & 22,00 & 510 & 56,50 & 89 & 9,80 & 1 & 0,1 & 904 & 100,00 \\
\hline Total & 471 & 3,90 & 1.622 & 13,50 & 8.668 & 72,20 & 1.172 & 9,80 & 75 & 0,6 & 12.008 & 100,00 \\
\hline
\end{tabular}

Fonte: Secretaria Municipal de Saúde do Município de São Luís 6,7; $\chi^{2}=230,766$; 5GL; $\mathrm{p}=0,000 ; \mathrm{n}=$ número de casos notificados de dengue clássica.

Tabela 3

Casos notificados de dengue clássica segundo sexo no Município de São Luís, Maranhão, Brasil, 1997-2002.

\begin{tabular}{lrrrrr}
\hline Ano & \multicolumn{2}{c}{ Masculino } & \multicolumn{2}{c}{ Sexo } & \multicolumn{2}{c}{ Total } \\
& $n$ & $\%$ & $n$ & $\%$ & \\
\hline 1997 & 2.156 & 50,20 & 2.139 & 49,80 & 4.295 \\
1998 & 2.723 & 49,50 & 2.778 & 50,50 & 5.501 \\
1999 & 220 & 42,90 & 293 & 57,00 & 513 \\
2000 & 101 & 52,10 & 93 & 47,90 & 194 \\
2001 & 278 & 46,30 & 323 & 53,70 & 601 \\
2002 & 407 & 45,00 & 497 & 55,00 & 904 \\
Total & 5.885 & 49,00 & 6.123 & 51,00 & 12.008 \\
\hline
\end{tabular}

Fonte: Secretaria Municipal de Saúde do Município de São Luís 6,7; $\chi^{2}=2,319$; $1 G L ; p=0,1278 ; n=$ número de casos de dengue clássica notificados.

\section{Tabela 4}

Índices de Infestação Predial e de Breteau (Aedes aegypti) e depósitos e imóveis (Aedes aegypti e Aedes albopictus) tratados no Município de São Luís, Maranhão, Brasil, 1997-2002.

\begin{tabular}{lccrr}
\hline Ano & $\begin{array}{c}\text { Índice de } \\
\text { Infestação } \\
\text { Predial }\end{array}$ & $\begin{array}{c}\text { Índice de } \\
\text { Breteau }\end{array}$ & Depósitos & Imóveis \\
\hline 1997 & 4,78 & 6,09 & 2.140 .025 & 630.787 \\
1998 & 7,15 & 8,18 & 2.538 .028 & 875.721 \\
1999 & 4,45 & 4,94 & 2.779 .919 & 1.089 .878 \\
2000 & 1,01 & 1,10 & 2.381 .002 & 1.052 .440 \\
2001 & 6,04 & 6,43 & 82.701 & 53.327 \\
2002 & 3,03 & 3,16 & 151.799 & 108.951 \\
Total & - & - & 9.921 .675 & 3.701 .153 \\
\hline
\end{tabular}

Fonte: Secretaria Municipal de Saúde do Município de São Luís 6,7. celos et al. 5, que já chamavam a atenção para essa situação, diferente daquela observada em outros estados, como o Rio de Janeiro, onde a incidência de FHD foi elevada 11. O motivo para tal comportamento ainda não foi compreendido, sendo necessários estudos mais aprofundados que venham oferecer uma resposta satisfatória para a questão.

O DS do Tirirical, com o maior número de casos de dengue, destacou-se por apresentar maior quantidade de criadouros potenciais; por ser uma área com grande demanda de prestação de serviços nos segmentos de borracharias, ferros-velhos e oficinas mecânicas, ambientes propícios ao desenvolvimento do Ae. aegyp$t i$; por apresentar muitos locais disponíveis para acúmulo de água 14 , predominando nesse caso os depósitos do tipo A (pneu); e pela disponibilidade e conseqüente facilidade com que são encontrados 15,16.

A incidência de casos de dengue predominou na faixa etária acima dos 15 anos, assemelhando-se aos achados de Gòmez-Dantés et al. 17, Vasconcelos et al. 5 e Nascimento et al. 18. A maior incidência da doença nas faixas etárias mais elevadas é um padrão observado em áreas indenes logo após a introdução de um sorotipo de vírus ${ }^{19}$. Em geral, esse padrão se modifica à medida que se instala o processo de endemização da doença 12.

Há estudos que demonstram maiores incidências de dengue em mulheres do que em homens 17,18; outros indicam transmissão similar entre os sexos 5 , como ocorreu neste trabalho.

As chuvas exercem grande influência na determinação do período de ocorrência da doença 20,21. Em São Luís, a situação estabelecida está diretamente relacionada com o aumento da precipitação pluviométrica e da umidade 
Número de depósitos positivos por tipo de mosquito encontrado em imóveis no Município de São Luís, Maranhão, Brasil, $1997-2002$.

\begin{tabular}{|c|c|c|c|c|c|c|c|c|c|c|c|c|c|c|c|c|}
\hline \multirow{2}{*}{$\begin{array}{l}\text { Tipos de } \\
\text { depó- } \\
\text { sitos a }\end{array}$} & \multicolumn{2}{|c|}{ e $\quad 1997$} & \multicolumn{2}{|c|}{1998} & \multicolumn{2}{|c|}{1999} & \multicolumn{2}{|c|}{2000} & \multicolumn{2}{|c|}{2001} & \multicolumn{2}{|c|}{2002} & \multicolumn{4}{|c|}{ Total } \\
\hline & $\begin{array}{c}\text { Ae. } \\
\text { aegypti }\end{array}$ & $\begin{array}{c}\text { Ae. albo- } \\
\text { pictus }\end{array}$ & $\begin{array}{c}\text { Ae. } \\
\text { aegypti }\end{array}$ & $\begin{array}{c}\text { Ae. albo- } \\
\text { pictus }\end{array}$ & Ae. & $\begin{array}{c}\text { Ae. albo- } \\
\text { pictus }\end{array}$ & $\begin{array}{c}\text { Ae. } \\
\text { aegypti }\end{array}$ & $\begin{array}{c}\text { Ae. albo- } \\
\text { pictus }\end{array}$ & - Ae. & $\begin{array}{c}\text { Ae. albo- } \\
\text { pictus }\end{array}$ & - $\begin{array}{c}\text { Ae. } \\
\text { aegypti }\end{array}$ & $\begin{array}{c}\text { Ae. albo- } \\
\text { pictus }\end{array}$ & - $\begin{array}{c}\text { Ae. } \\
\text { aegypti }\end{array}$ & $\%$ & $\begin{array}{c}\text { Ae. albo- } \\
\text { pictus }\end{array}$ & $\%$ \\
\hline A & 131 & 52 & 357 & 51 & 404 & 88 & 895 & 94 & 1.658 & 120 & 974 & 65 & 4.419 & 3,30 & 470 & 13,30 \\
\hline$B$ & 3.171 & 206 & 8.645 & 207 & 9.787 & 348 & 6.855 & 171 & 46.444 & 580 & 32.787 & 327 & 107.689 & 80,00 & 1.839 & 52,10 \\
\hline C & 186 & 22 & 508 & 23 & 575 & 37 & 456 & 30 & 3.191 & 56 & 1.399 & 31 & 6.315 & 4,70 & 199 & 5,60 \\
\hline$D$ & 36 & 8 & 97 & 7 & 110 & 13 & 271 & 16 & 436 & 20 & 276 & 6 & 1.226 & 0,90 & 70 & 2,00 \\
\hline$E$ & 143 & 56 & 389 & 54 & 440 & 94 & 675 & 88 & 2.100 & 142 & 1.059 & 67 & 4.806 & 3,60 & 501 & 14,20 \\
\hline $\mathrm{F}$ & 28 & 4 & 76 & 6 & 86 & 7 & 44 & 3 & 428 & 11 & 275 & 8 & 937 & 0,70 & 39 & 1,10 \\
\hline G & 107 & 8 & 292 & 8 & 330 & 13 & 248 & 5 & 1.689 & 23 & 1.014 & 15 & 3.680 & 2,70 & 72 & 2,00 \\
\hline $\mathrm{H}$ & 8 & 2 & 22 & 2 & 24 & 4 & 17 & 3 & 112 & 7 & 43 & 3 & 226 & 0,20 & 21 & 0,60 \\
\hline 1 & 111 & 28 & 301 & 28 & 342 & 49 & 434 & 51 & 1.737 & 61 & 825 & 43 & 3.750 & 2,80 & 260 & 7,40 \\
\hline $\mathrm{J}$ & 2 & 1 & 2 & 1 & 3 & 0 & 1 & 0 & 14 & 0 & 17 & 0 & 39 & 0,00 & 2 & 0,00 \\
\hline K & 41 & 7 & 119 & 7 & 133 & 12 & 163 & 5 & 795 & 26 & 293 & 4 & 1.544 & 1,10 & 61 & 1,70 \\
\hline Total & 3.964 & 394 & 10.808 & 394 & 12.234 & 665 & 10.059 & 466 & 58.604 & 1.046 & 38.962 & 569 & 134.631 & 100,00 & 3.534 & 100,00 \\
\hline
\end{tabular}

Tipos de depósitos: $\mathrm{A}$ = pneu; $\mathrm{B}$ = tambor/tanque/barril/tina/tonel/depósito de barro; $\mathrm{C}$ = vaso de planta;

$D=$ material de construção/peça de carro; $E$ = garrafa/lata/plástico; $F=$ poço/cisterna/cacimba; $G=$ caixa d'água;

$\mathrm{H}=$ recipiente natural; $\mathrm{I}=$ outros; $\mathrm{J}=$ armadilha; $\mathrm{K}=$ pool

relativa do ar. Esses fatores abióticos não só aumentam consideravelmente a quantidade de criadouros disponíveis para o desenvolvimento das formas imaturas do vetor, incluindo-se aí os depósitos naturais e artificiais de água, como também geram condições ambientais para o desenvolvimento dos adultos 22 .

Analisando-se os resultados dos trabalhos de campo do Programa de Controle da Febre Amarela e Dengue (PCFAD) de São Luís, referentes aos depósitos preferenciais do Ae. aegypti e do Ae. albopictus, observaram-se situações similares às encontradas em outras cidades 14,23, destacando-se os depósitos do tipo B por proporcionar condições ideais à reprodução do vetor. Cabe lembrar que, em áreas onde se registra baixo índice pluviométrico e/ou suprimento de água deficiente, a população precisa armazená-la para suas necessidades básicas 24 .

Chiaravalloti-Neto 25 afirma que a manutenção de plantas em vasos com água e a utilização de vasos com pratos, onde ocorre armazenamento do líquido, concorrem como fator importante nos níveis de infestação dos vetores, despontando como importantes criadouros dos mosquitos da dengue.

Chama-se a atenção para a presença marcante do Ae. albopictus em nosso meio que, a exemplo de São José do Rio Preto, São Paulo 26, vem ocupando quase todas as áreas urbanas, mostrando o seu poder de difusão, pois sua existência tem sido associada a ambientes silvestres 27,28. Apesar de Forattini et al. 29 descar- tarem sua importância epidemiológica, é necessário elaborar modelos teóricos consistentes que forneçam mais informações sobre a sua biologia.

No Município de São Luís não existe nenhum estudo demonstrando a importância epidemiológica dessa espécie ou sua presença também no ambiente silvestre. Diante de tais possibilidades é preciso estar alerta, visto ser um vetor potencial não só da dengue, como também da febre amarela e outras arboviroses.

Os índices elevados do IIP e do IB apresentados neste estudo, não obstante o número de depósitos e imóveis tratados ao longo do período estudado, colocam São Luís numa situação de grande vulnerabilidade, tendo em vista os índices usados para caracterizar situação de iminente perigo à saúde pública pela presença do transmissor da dengue, se uma localidade apresenta IIP > 1 e IB > 5 8,30.

Em muitos outros países 31, assim como nos diversos estados brasileiros, as ações de combate ao Aedes vêm apresentando baixa efetividade, devido à complexidade da biologia desse vetor e à sua capacidade de adaptação ao ambiente humano. Em São Luís, as dificuldades também são operacionais: aumento do número de imóveis em conseqüência da ocupação desordenada; capacitação inadequada do pessoal de campo; dificuldades de operacionalização na execução das ações de campo; quebra no processo de rotina das supervisões; freqüente rotatividade dos convênios; interrupção fre- 
qüente do trabalho do UBV (ultrabaixo volume); indisponibilidade de recursos orçamentários e financeiros; grande número de pendências por causa de imóveis fechados; dificuldade de manter a articulação com outros segmentos institucionais; dificuldade de estabelecer parceria de serviço com os municípios limítrofes; e atraso no repasse para a Secretaria Municipal de Saúde do Município de São Luís do insumo cipermetrina, para tratamento perifocal em pontos estratégicos.

Em síntese, o quadro desenhado nos anos de 1997 e 1998 mostra uma correlação com a casuística da doença na região nordeste e no Brasil, quando se constatam elevadas taxas de incidência da endemia. De 1999 a 2000 observa-se um período compatível com uma pós-epidemia, quando há certa acomodação no nú-

\section{Resumo}

Foram notificados 12.008 casos de dengue no período de 1997 a 2002, com picos em 1997 (35,80\%) e 1998 (45,80\%). A doença predominou na faixa etária de 1549 anos $(72,20 \%)$ e na estação chuvosa (83,30\%), correlacionando-se positivamente com a precipitação pluviométrica $(r=0,84)$ e a umidade relativa $(r=0,76)$, e negativamente com a temperatura $(r=-0,78)$. Não houve diferença significativa $\left(\chi^{2}=2,319 ; p>0,05\right)$ no número de casos entre os sexos masculino $(49,00 \%) e$ feminino (51,00\%). Os sete distritos sanitários resultaram positivos: Tirirical (27,00\%), Coroadinho (17,00\%), Bequimão (16,00\%), Cohab (14,00\%), Itaqui-Bacanga (11,00\%), Centro (8,00\%) e Vila Esperança (7,00\%). O indice de infestação predial oscilou de 1,01 a 7,15 e o indice de Breteau, de 1,10 a 8,18. Os depósitos mais freqüentes foram: tambor e similares (80,00\%), vaso de planta (4,70\%), garrafa, lata e plástico (3,60\%), pneu (3,30\%) e caixa d'água (2,70\%); os demais representaram 5,7\%. Para Ae. aegypti foram encontrados 134.631 depósitos positivos, predominando os do tipo $B(80,00 \%)$, seguidos pelos dos tipos $C$ (4,70\%), E (3,60\%) e A (3,30\%).

Dengue; Aedes; Distritos Sanitários

\section{Colaboradores}

V. S. Gonçalves Neto participou da coleta, da organização e da análise dos dados. J. M. M. Rebêlo colaborou na análise dos dados e na redação do artigo. Os autores em conjunto realizaram a redação do artigo. mero de casos. A doença apresenta um padrão sazonal, com maior incidência de casos na estação chuvosa. A casuística da doença oscilou de acordo com o IIP e o IB no período em estudo. Pelos tipos de criadouros/depósitos com água encontrados, é necessário manter uma contínua atividade educativa de esclarecimento à população sobre o risco de proliferação doméstica do vetor. As condições precárias de saneamento básico e a concentração da população de baixa renda em áreas periféricas das grandes cidades criam um ambiente favorável ao desenvolvimento e à proliferação do $A e$. $a e$ gypti e do Ae. albopictus, daí a necessidade urgente de uma participação efetiva da sociedade na implementação de medidas práticas objetivando a redução dos potenciais criadouros de mosquitos.

\section{Agradecimentos}

À equipe da Superintendência de Vigilância Epidemiologia e Sanitária da Secretaria Municipal de Saúde de São Luís e à Vigilância Epidemiológica da Secretaria Extraordinária de Saúde Preventiva do Estado.

\section{Referências}

1. Fundação Nacional de Saúde, Ministério da Saúde. Boletim Epidemiológico 1999; Ano III (Edição Especial)

2. Fundação Nacional de Saúde, Ministério da Saúde. Boletim Eletrônico Epidemiológico 2001; Ano 01 no 01 .

3. Silveira AC. Dengue: aspectos epidemiológicos e de controle. Rev Soc Bras Med Trop 1998; 31 Suppl 2:5-14.

4. Fundação Nacional de Saúde, Ministério da Saúde. Boletim Eletrônico Epidemiológico 2002; Ano 02 no 02.

5. Vasconcelos PFC, Lima JWO, Raposo ML, Rodrigues SG, da Rosa JST, Amorim SMC, et al. Inquérito soro-epidemiológico na Ilha de São Luís durante epidemia de dengue no Maranhão. Rev Soc Bras Med Trop 1999; 32:171-9.

6. Secretaria Municipal de Saúde do Município de São Luís. Relatório anual de atividades, período: 2002. São Luís: Secretaria Municipal de Saúde do Município de São Luís; 2003.

7. Secretaria Municipal de Saúde do Município de São Luís. Relatório quadrienal de atividades, período: 1997 a 2000. São Luís: Secretaria Municipal 
de Saúde do Município de São Luís; 2001.

8. Fundação Nacional de Saúde, Ministério da Saúde. Programa Nacional de Controle da Dengue PNCD. Brasília: Fundação Nacional de Saúde, Ministério da Saúde; 2002.

9. Fundação Instituto Brasileiro de Geografia e Estatística. Anuário estatístico do Brasil. Rio de Janeiro: Fundação Instituto Brasileiro de Geografia e Estatística; 2002.

10. Vasconcelos PFC, Lima JWO, da Rosa APAT, Timbó MJ, da Rosa EST, Lima HR, et al. Epidemia de dengue em Fortaleza, Ceará: inquérito soro-epidemiológico aleatório. Rev Saúde Pública 1998; 32:447-54.

11. Nogueira RMR, Miagostovich MP, Schatzmayr JG, Santos FB, Araújo ESM, Filipps AMB, et al. Dengue in the State of Rio de Janeiro, Brazil, 19861998. Mem Inst Oswaldo Cruz. Rio de Janeiro 1999; 94:297-304.

12. Teixeira MG, Costa MCN, Barreto ML, Barreto FR. Epidemiologia do dengue em Salvador-Bahia, 1995-1999. Rev Soc Bras Med Trop 2001; 34:269-74.

13. Halstead SB. The Alexander D. Langmuir lecture. The pathogenesis of dengue. Molecular epidemiology in infectious disease. Am J Epidemiol 1991; 114:632-48.

14. Medeiros MNL, Medeiros RNS. Controle de dengue 1996 a 1998 - saneamento ambiental em São Luís. In: Anais do IX Simpósio Luso-Brasileiro de Engenharia Sanitária e Ambiental; 2000 Abr 9-14; Porto Seguro, Bahia. [CD-ROM].

15. Focks DA, Sackett SR, Bailey DL, Dame DA. Observations on container-breeding mosquitoes in New Orleans, Louisiana, with an estimate of the population density of Aedes aegypti (L.). Am J Trop Med Hyg 1981; 30:1329-35.

16. Lopes J, Silva MAN, Borsato AM, de Oliveira VDRB, Oliveira FJA. Aedes (Stegomyia) aegypti L. e a culicideofauna associada em área urbana da região sul, Brasil. Rev Saúde Pública 1993; 27:326-33.

17. Gòmez-Dantés H, Montesano-Castellanos R, Lopez-Moreno S, Tapia-Conyer R. El dengue en México. Situación epidemiológica reciente. Gac Med Mex 1995; 131:237-40.

18. Nascimento DMB, Coelho RN, Rodrigues SG. Diagnóstico laboratorial da dengue no município de Belém - Pará: a atuação do Laboratório Central do Estado do Pará. Rev Soc Bras Med Trop 2003; 36 Suppl 1:484-5.

19. Gubler DJ. Dengue and dengue hemorrhagic fever: its history and resurgence as a global health problem. In: Gubler DJ, Kuno G, editors. Dengue and dengue hemorrhagic fever. New York: CAB International; 1997. p. 1-22.

20. Rebêlo JMM, Costa JML, Silva FS, Pereira YNO, Silva JM. Distribuição de Aedes aegypti e do dengue no Estado do Maranhão, Brasil. Cad Saúde Pública 1999; 15:477-86.
21. Sandoval JJF, Marreiro LS, Saraiva MGG, Alecrim WD, Guerra MVF, Albuquerque BC, et al. Controle do dengue na cidade de Manaus - Amazonas, 2001-2002. Rev Soc Bras Med Trop 2003; 36 Suppl $1: 490$.

22. Glasser CM, Gomes AC. Clima e sobreposição da distribuição de Aedes aegypti e Aedes albopictus na infestação do Estado de São Paulo. Rev Saúde Pública 2002; 36:166-72.

23. Pinheiro VCS, Tadei WP. Frequency, diversity, and productivity study on the Aedes aegypti most preferred containers in the city of Manaus, Amazonas, Brazil. Rev Inst Med Trop S Paulo 2002; 44:245-50.

24. Barcellos C, Coutinho K, Pina MF, Magalhães MMAF, Paola JCMD, Santos SM. Inter-relacionamento de dados ambientais e de saúde: análise de risco à saúde aplicada ao abastecimento de água no Rio de Janeiro utilizando Sistemas de Informações Geográficas, Cad Saúde Pública 1998; 14:597-605.

25. Chiaravalloti-Neto F. Conhecimentos da população sobre dengue, seus vetores e medidas de controle em São José do Rio Preto, São Paulo. Cad Saúde Pública 1997; 13:447-53.

26. Chiaravalloti-Neto F, Dibo MR, Barbosa AAC, Battigaglia M. Aedes albopictus (S) na região de São José do Rio Preto, SP: estudo da sua infestação em área já ocupada pelo Aedes aegypti e discussão de seu papel como possível vetor de dengue e febre amarela. Rev Soc Bras Med Trop 2002; 35:351-7.

27. Neves DP, Silva RF. Aspectos da biologia do Aedes albopictus (Skuse, 1894) (Diptera: Culicidae), em nível de campo. Mem Inst Oswaldo Cruz 1989; 84:403-4.

28. Gomes AC, Forattini OP, Kakitani I, Marques GRAM, Marques CCA, Marucci D, et al. Microhabitats de Aedes albopictus (Skuse) na região do Vale do Paraíba, Estado de São Paulo, Brasil. Rev Saúde Pública 1992; 26:108-18.

29. Forattini OP, Marques GRAM, Kakitani I, Brito M, Sallum MAM. An unusual ground larval habitat of Aedes albopictus. Rev Inst Med Trop S Paulo 1998; 40:121-2.

30. Gomes AC. Medidas dos níveis de infestação urbana para Aedes (Stegomyia) aegypti e Aedes (Stegomyia) albopictus em programa de vigilância entomológica. Inf Epidemiol SUS 1998; 7:49-57.

31. Goh KT. Changing epidemiology of dengue in Singapore. Lancet 1995; 346:1098.

Recebido em 09/Jan/2004

Versão final reapresentada em 07/Mai/2004

Aprovado em 10/Mai/2004 